\title{
GELFAND THEORY OF PSEUDO DIFFERENTIAL OPERATORS AND HYPOELLIPTIC OPERATORS
}

\author{
BY \\ MICHAEL E. TAYLOR
}

\begin{abstract}
This paper investigates an algebra $\mathfrak{U}$ of pseudo differential operators generated by functions $a(x) \in C^{\infty}\left(R^{n}\right) \cap L^{\infty}\left(R^{n}\right)$ such that $D^{\alpha} a(x) \rightarrow 0$ as $|x| \rightarrow \infty$, if $|\alpha| \geqq 1$, and by operators $q(D) Q^{-1 / 2}$ where $q(D)<P(D), Q=I+P(D)^{*} P(D)$, and $P(D)$ is hypoelliptic. It is proved that such an algebra has compact commutants, and the maximal ideal space of the commutative $C^{*}$ algebra $\mathfrak{A} / J$ is investigated, where $J$ consists of the elements of $\mathfrak{A}$ which are compact. This gives a necessary and sufficient condition for a differential operator $q(x, D): \mathfrak{B}_{2, \tilde{P}} \rightarrow L^{2}$ to be Fredholm. (Here and in the rest of this paragraph we assume that the coefficients of all operators under consideration satisfy the conditions given on $a(x)$ in the first sentence.) It is also proved that if $p(x, D)$ is a formally selfadjoint operator on $R^{n}$ which has the same strength as $P(D)$ uniformly on $R^{n}$, then $p(x, D)$ is selfadjoint, with domain $\mathfrak{B}_{2, \tilde{P}}\left(R^{n}\right)$, and semibounded, if $n \geqq 2$. From this a Gårding type inequality for uniformly strongly formally hypoelliptic operators and a global regularity theorem for uniformly formally hypoelliptic operators are derived. The familiar local regularity theorem is also rederived.

It is also proved that a hypoelliptic operator $p(x, D)$ of constant strength is formally hypoelliptic, in the sense that for any $x_{0}$, the constant coefficients operator $p\left(x_{0}, D\right)$ is hypoelliptic.
\end{abstract}

Introduction. In [2], H. O. Cordes and E. A. Hermann investigate several algebras of pseudo differential operators. The first section of this paper considers an algebra similar to one of theirs, but related to general hypoelliptic operators with constant coefficients rather than to the special case of the Laplacian. The second section applies these results to obtain some spectral and regularity theorems for uniformly formally hypoelliptic differential operators with constant strength on $R^{n}$, with smooth coefficients which are suitably restricted at infinity. In the third section we introduce a couple of algebras of pseudo differential operators, for which the considerations of §II remain valid, and investigate the existence and nature of parametrices for elements of this algebra. §IV is devoted to a result of a different nature, namely that every hypoelliptic differential operator of constant strength is formally hypoelliptic. This provides a converse to the classical theorem of Hörmander [6] and Malgrange [7] to the effect that a formally hypoelliptic differential operator of constant strength is hypoelliptic.

Received by the editors February 19, 1970.

AMS 1969 subject classifications. Primary 3523; Secondary 3548, 4765.

Key words and phrases. Pseudo differential operator, hypoelliptic operator, Fredholm operator.

Copyright (C) 1971, American Mathematical Society 
Notations used in the paper, for differential operators, e.g. $P(D)$, and spaces of distributions, e.g. $\mathfrak{B}_{2, \tilde{P}}\left(R^{n}\right)$, will be that of Hörmander [4], with only two exceptions. First, we shall take $p(x, D)^{t}$ to be the formal adjoint of $p(x, D)$ with respect to an inner product $($,$) which is linear in the first argument and conjugate linear in the$ second. Also, in §III, we define $H^{s}\left(R^{n}\right)=\mathfrak{B}_{2, k_{s}}\left(R^{n}\right)$ with $k_{s}(\xi)=\left(1+|P(\xi)|^{2}\right)^{s / 2}$ and $P(D)$ the hypoelliptic operator with constant coefficients which happens to be under consideration.

This paper is part of the author's Ph.D. dissertation, written under the direction of Professor H. O. Cordes, whose guidance is gratefully acknowledged.

I. Gelfand theory of pseudo differential operators. Let $P(D)$ be a hypoelliptic differential operator with constant coefficients. We consider the $C^{*}$ algebra $\mathfrak{A}$ of operators on $L^{2}\left(R^{n}\right)$ generated by

(i) $a(x), a \in C^{\infty}\left(R^{n}\right) \cap L^{\infty}\left(R^{n}\right), D^{\alpha} a(x) \rightarrow 0$ as $|x| \rightarrow \infty$ if $|\alpha| \neq 0$;

(ii) $q(D) Q^{-1 / 2}$ where $Q=I+P(D)^{t} P(D)$, where $q(D)<P(D)$, and also $\varphi(D)$ $=F^{-1} \varphi(x) F$ where $\varphi \in C_{0}^{\infty}\left(R^{n}\right)$ and $F$ is the Fourier transform. If $J$ is the ideal of compact operators belonging to $\mathfrak{A}$, we shall show that $\mathfrak{A} / J$ is commutative and shall exhibit its maximal ideal space. From this we shall deduce information on Fredholm operators on $L^{2}\left(R^{n}\right)$ and also on certain other spaces $\mathfrak{B}_{2, k}\left(R^{n}\right)$.

Our first proposition follows closely the proof of Lemma 4 in [2].

Proposition 1. If $a(x)$ is as in (i) above and $t>0$, then $\left[a, Q^{-t}\right]=C Q^{-t}$ for some compact operator $C$ (depending on $t$ ).

Proof. We write the integral formula $2 \pi i\left[a, Q^{-t}\right]=\int_{\gamma} \lambda^{-t}\left[a, R_{\lambda}\right] d \lambda$, where $R_{\lambda}$ $=(\lambda-Q)^{-1}$ and $\gamma$ is the line from $\frac{1}{2}-i \infty$ to $\frac{1}{2}+i \infty$. To evaluate this, we note that

Thus

$$
\begin{aligned}
{\left[a, R_{\lambda}\right] } & =a R_{\lambda}-R_{\lambda} a=R_{\lambda}(\lambda-Q) a R_{\lambda}-R_{\lambda} a(\lambda-Q) R_{\lambda} \\
& =R_{\lambda}[\lambda-Q, a] R_{\lambda}=R_{\lambda}[a, Q] R_{\lambda} \\
& =-R_{\lambda}\left\{\sum_{\alpha \neq 0} \frac{1}{\alpha !}\left(D^{\alpha} a\right) Q^{(\alpha)}(D)\right\} R_{\lambda} .
\end{aligned}
$$

$$
\begin{aligned}
2 \pi i[ & \left.a, Q^{-t}\right] \\
= & \int_{\gamma} \lambda^{-t}\left[a, R_{\lambda}\right] d \lambda \\
= & \sum_{\alpha \neq 0} \frac{1}{\alpha !} \int_{\gamma} \lambda^{-t} R_{\lambda}\left(D^{\alpha} a\right) Q^{(\alpha)}(D) R_{\lambda} d \lambda \\
= & -\sum_{\alpha \neq 0}\left(D^{\alpha} a\right) Q^{(\alpha)}(D) \frac{1}{\alpha !} \int_{\gamma} \lambda^{-t} R_{\lambda}^{2} d \lambda+\sum_{\alpha \neq 0} \frac{1}{\alpha !} \int_{\gamma} \lambda^{-t}\left[D^{\alpha} a, R_{\lambda}\right] Q^{(\alpha)}(D) R_{\lambda} d \lambda \\
= & -\sum_{\alpha \neq 0} \frac{1}{\alpha !}\left(D^{\alpha} a\right) Q^{(\alpha)}(D) \int_{\gamma} \lambda^{-t} R_{\lambda}^{2} d \lambda \\
& -\sum_{\alpha_{1}, \alpha_{2} \neq 0} \int_{\gamma} \frac{1}{\alpha_{1} ! \alpha_{2} !} \lambda^{-t} R_{\lambda}\left(D^{\left.\alpha_{1}+\alpha_{2} a\right) Q^{\left(\alpha_{1}\right)}(D) Q^{\left(\alpha_{2}\right)}(D) R_{\lambda}^{2} d \lambda}\right.
\end{aligned}
$$




$$
\begin{aligned}
& =-\sum_{\alpha \neq 0} \frac{1}{\alpha !}\left(D^{\alpha} a\right) Q^{(\alpha)}(D)\left(\begin{array}{c}
-t \\
1
\end{array}\right) Q^{-t-1} \\
& -\sum_{\alpha_{1}, \alpha_{2} \neq 0}\left(D^{\left.\alpha_{1}+\alpha_{2} a\right)} Q^{\left(\alpha_{1}\right)}(D) Q^{\left(\alpha_{2}\right)}(D)\left(\begin{array}{c}
-t \\
2
\end{array}\right) Q^{-t-2} \frac{1}{\alpha_{1} ! \alpha_{2} !}\right. \\
& +\sum_{\alpha_{1}, \alpha_{2} \neq 0} \frac{1}{\alpha_{1} ! \alpha_{2} !} \int_{\gamma} \lambda^{-t}\left[D^{\alpha_{1}+\alpha_{2}} a, R_{\lambda}\right] Q^{\left(\alpha_{1}\right)}(D) Q^{\left(\alpha_{2}\right)}(D) R_{\lambda}^{2} d \lambda \\
& =\cdots \text {. } \\
& =-\sum_{j=1}^{m-1} \sum_{\alpha_{1}, \ldots, \alpha_{j} \neq 0} \frac{1}{\alpha_{1} ! \cdots \alpha_{j} !}\left(\begin{array}{c}
-t \\
j
\end{array}\right)\left(D^{\left.\alpha_{1}+\cdots+\alpha_{j} a\right)} Q^{\left(\alpha_{1}\right)}(D) Q^{-1} \cdots Q^{\left(\alpha_{j}\right)}(D) Q^{-1} Q^{-t}\right. \\
& -\sum_{\alpha_{1}, \ldots, \alpha_{m} \neq 0} \frac{1}{\alpha_{1} ! \cdots a_{m} !} \int_{\gamma} \lambda^{-t} R_{\lambda}\left(D^{\alpha_{1}+\cdots+\alpha_{m}} a\right) R_{\lambda}^{m} d \lambda Q^{\left(\alpha_{1}\right)}(D) \cdots Q^{\left(\alpha_{m}\right)}(D) .
\end{aligned}
$$

The first sum is clearly of the form $C Q^{-t}$ where $C$ is compact, since $b(x) \varphi(D)$ is compact provided $b(x)$ and $\varphi(\xi)$ vanish at infinity. The integral in the second sum is equal to

$$
\int_{\gamma}\left(\lambda^{-t} R_{\lambda} Q^{r}\right)\left(Q^{-r}\left(D^{\left.\alpha_{1}+\cdots+\alpha_{m} a\right)}\right)\left(R_{\lambda} Q\right)^{m} d \lambda Q^{\left(\alpha_{1}\right)}(D) Q^{-(1-s)} \cdots Q^{\left(\alpha_{m}\right)}(D) Q^{-(1-s)} Q^{-m s} .\right.
$$

If $0<r<t$ for $t \leqq 1$ and $r=1$ when $t>1$, we see that $\left\|\lambda^{-t} R_{\lambda} Q^{r}\right\| \leqq C(1+|\lambda|)^{-1-\varepsilon}$ for some $\varepsilon>0$, that $Q^{-r}\left(D^{\alpha_{1}+\cdots+\alpha_{m}} a\right)$ is compact and independent of $\lambda$, and that $\left\|\left(R_{\lambda} Q\right)^{m}\right\|<C^{\prime}$. It remains to show that there is an $s>0$ such that $\left|Q^{(\alpha)}(\xi)\right| /|Q(\xi)|^{1-s}$ is bounded when $\alpha \neq 0$. For then we just pick $m$ so large that $m s \geqq t$. But since $Q$ is hypoelliptic, we have $\left|Q^{(\alpha)}(\xi)\right| \leqq C(1+|\xi|)^{-\rho|\alpha|}|Q(\xi)|$, some $\rho>0$, so $\left|Q^{(\alpha)}(\xi)\right| /|Q(\xi)|^{1-s} \leqq C(1+|\xi|)^{-\rho|\alpha|}|Q(\xi)|^{s}<C^{\prime} \quad$ when $\alpha \neq 0$ if $|Q(\xi)|^{s} \leqq$ $C(1+|\xi|)^{\rho|\alpha|}$ which follows if $s \leqq \rho / \nu$ where $\nu$ is the order of $Q(\xi)$. The proof is complete.

Before we show that $\mathfrak{A}$ has compact commutators, we need some information on the behavior of $\left|q^{(\alpha)}(\xi)\right| / Q(\xi)^{1 / 2}$ for large $\xi$, or equivalently, the behavior of $\left|q^{(\alpha)}(\xi)\right| /|P(\xi)|$. The result we give also implies that, if $q(D)<P(D)$, then $q(\xi) / P(\xi) \in S_{\rho, 0}^{0}$, in the notation of [5].

LEMMA 1. Suppose $P_{1}(\xi)$ is a hypoelliptical polynomial such that $\left|P_{1}^{(\alpha)}(\xi) / P_{1}(\xi)\right|$ $\leqq C(1+|\xi|)^{-\rho|\alpha|}$ for large $\xi$. If $q(\xi) / P_{1}(\xi)$ is bounded for large $\xi$, then $\left|q^{(\beta)}(\xi) / P_{1}(\xi)\right|$ $\leqq C^{\prime}(1+|\xi|)^{-\rho|\beta|}$ for large $\xi$.

Proof. We can choose a nonzero number $a$ sufficiently small that $P_{1}(D)$ and $P_{1}(D)+a q(D)=P_{2}(D)$ have the same strength. Then $P_{2}(D)$ is hypoelliptic by Theorem 4.1.6 of [4] and if $d_{j}(\xi)$ is the distance from $\xi \in R^{n}$ to the surface $\left\{\zeta \in C^{n}: P_{j}(\zeta)=0\right\}$, we have $1 / b \leqq d_{1}(\xi) / d_{2}(\xi) \leqq b$ for $\xi$ large, for some constant $b$. From the inequality $1 / d \leqq d_{j}(\xi) \sum_{\alpha \neq 0}\left|P_{j}^{(\alpha)}(\xi) / P_{j}(\xi)\right|^{1 /|\alpha|} \leqq d, \quad \xi \in R^{n}, \quad|\xi|>R$, which is Lemma 4.1 .1 of [4], we see that the hypothesis yields $d_{1}(\xi) \geqq C_{1}|\xi|^{-\rho}$, so $d_{2}(\xi) \geqq C_{2}|\xi|^{-\rho}$, and hence $\left|P_{2}^{(\alpha)}(\xi) / P_{2}(\xi)\right| \leqq C_{3}|\xi|^{-\rho|\alpha|}$. 
Now, for large $\xi$,

$$
\begin{aligned}
\left|\frac{q^{(\beta)}(\xi)}{P_{1}(\xi)}\right| & =\left|\frac{1}{a}\right|\left|\frac{P_{2}^{(\rho)}(\xi)-P_{1}^{(\beta)}(\xi)}{P_{1}(\xi)}\right| \leqq\left|\frac{1}{a}\right|\left|\frac{P_{2}^{(\beta)}(\xi)}{P_{2}(\xi)}\right|\left|\frac{P_{2}(\xi)}{P_{1}(\xi)}\right|+\left|\frac{1}{a}\right|\left|\frac{P_{1}^{(\beta)}(\xi)}{P_{1}(\xi)}\right| \\
& \leqq C^{\prime}|\xi|^{-\rho|\beta|} .
\end{aligned}
$$

We record the following corollary here, although we shall make no further use of it.

CoRollary. If $P_{1}(\xi)$ and $q(\xi)$ are as above, then $q(\xi) / P_{1}(\xi) \in S_{\rho, 0}^{0}$.

Proof. Using the above lemma it is easy to obtain the desired estimate on $D^{\alpha}\left(q(\xi) / P_{1}(\xi)\right)$.

Proposition 2. Let $a(x)$ be as in (i), $q(D)<P(D)$. If $\varphi(D)=q(D) Q^{-1 / 2}$ or if $\varphi(D)=F^{-1} \varphi(\xi) F$ with $\varphi \in C_{0}^{\infty}$, then $[a, \varphi(D)]$ is compact. Hence $\mathfrak{X} / J$ is commutative.

Proof. If $\varphi(D)=q(D) Q^{-1 / 2}$, we have

$$
\begin{array}{rlrl}
a \varphi(D)-\varphi(D) a= & a q(D) Q^{-1 / 2}-q(D) Q^{-1 / 2} a & \\
= & a Q^{-1 / 2} q(D)-Q^{-1 / 2} a q(D)+Q^{-1 / 2} a q(D)-Q^{-1 / 2} q(D) a \\
= & {\left[a, Q^{-1 / 2}\right] q(D)+Q^{-1 / 2}[a, q(D)]} & \\
= & C Q^{-1 / 2} q(D)-Q^{-1 / 2} \sum_{\alpha \neq 0} \frac{1}{\alpha !}\left(D^{\alpha} a\right) q^{(\alpha)}(D), & C \text { compact } \\
= & C^{\prime}\left(q(D) Q^{-1 / 2}\right)-\sum_{\alpha \neq 0} \frac{1}{\alpha !}\left(D^{(\alpha)} a\right)\left(q^{(\alpha)}(D) Q^{-1 / 2}\right), & C^{\prime} \text { compact } \\
& +\sum_{\alpha \neq 0} C_{\alpha}\left(q^{(\alpha)}(D) Q^{-1 / 2}\right) &
\end{array}
$$

The first term is compact since $q(D) Q^{-1 / 2}$ is bounded on $L^{2}$. The second term is compact since $\left|q^{(\alpha)}(\xi)\right| / Q(\xi)^{1 / 2} \leqq C(1+|\xi|)^{-\rho|\alpha|}$ and $D^{\alpha} a$ vanishes at infinity, when $\alpha \neq 0$. On the other hand, if $\varphi \in C_{0}^{\infty}$, then $\varphi(\xi)$ can be approximated uniformly on $R^{n}$ by an element of the algebra generated by $\left\{q(\xi) / Q_{1}(\xi)^{1 / 2}: q(D)<P_{1}(D)\right\}$ with $Q_{1}(\xi)=1+\left|P_{1}(\xi)\right|^{2}$ and $P_{1}(\xi)=|\xi|^{2}$, so the compactness of $[a, \varphi(D)]$ in this case follows from the result just proved. That $\mathfrak{A} / J$ is commutative is now obvious.

We now determine the maximal ideal space of $\mathfrak{U} / J$. (We shall identify the maximal ideal space of a commutative Banach algebra with unit with the space of homomorphisms of the algebra onto the complex numbers.) Let $\mathfrak{A}_{1}$ be the $C^{*}$ algebra generated by the $a(x)$ as in (i) of the beginning of this section, $\mathfrak{A}_{2}$ the $C^{*}$ algebra generated by the operators as in (ii). Then we have maps $\alpha_{j}: \mathfrak{A}_{j} \rightarrow \mathfrak{A} / J$ and their duals give maps of maximal ideal spaces

$$
\mathscr{M} \stackrel{\alpha_{1}^{t} \times \alpha_{2}^{t}}{\longrightarrow} \mathscr{M}_{1} \times \mathscr{M}_{2},
$$

where $\mathscr{M}$ is the maximal ideal space of $\mathfrak{A} / J$ and $\mathscr{M}_{j}$ is the maximal ideal space of $\mathfrak{A}_{j}$. Note that this map of $\mathscr{M} \rightarrow \mathscr{M}_{1} \times \mathscr{M}_{2}$ is $1-1$. For if $m, m^{\prime} \in \mathscr{M}$, with $\alpha_{j}^{t}(m)$ 
$=\alpha_{j}^{t}\left(m^{\prime}\right), j=1,2$, we see that $A_{1} \in \mathfrak{A}_{1}$ implies $\left\langle\alpha_{1} A_{1}, m\right\rangle=\left\langle\alpha_{1} A_{1}, m^{\prime}\right\rangle$ and $A_{2} \in \mathfrak{A}_{2}$ implies $\left\langle\alpha_{2} A_{2}, m\right\rangle=\left\langle\alpha_{2} A_{2}, m^{\prime}\right\rangle$, and since $m$ and $m^{\prime}$ are continuous and multiplicative it follows that $\langle A, m\rangle=\left\langle A, m^{\prime}\right\rangle$ for all $A \in \mathfrak{A} / J$ since $\mathfrak{A}_{1}$ and $\mathfrak{A}_{2}$ generate 2l. Thus $m=m^{\prime}$. Thus we must only identify $\mathscr{M}$ as a subspace of $\mathscr{M}_{1} \times \mathscr{M}_{2}$.

In the case here, $\mathscr{M}_{1}$ is the smallest compactification of $R_{x}^{n}$ to which all functions $a(x)$ as in (i) extend continuously and $\mathscr{M}_{2}$ is the smallest compactification of $R_{\xi}^{n}$ to which all functions $q(\xi) / Q(\xi)^{1 / 2}$ with $q(D)$ weaker than $P(D)$ extend continuously. (A couple of times it will be convenient to identify $\mathscr{C}\left(\mathscr{M}_{j}\right)$, the space of continuous functions on $\mathscr{M}_{j}$, with the space of continuous functions on $R^{n}$ which can be so extended.) We have the natural inclusion $R_{x}^{n} \times R_{\xi}^{n} \subset \mathscr{M}_{1} \times \mathscr{M}_{2}$.

In order to determine $\mathscr{M}$ as a subset of $\mathscr{M}_{1} \times \mathscr{M}_{2}$, we make use of the following lemma, due to H. O. Cordes (unpublished), whose proof we include here for completeness.

Lemma 2. Suppose $f \in L^{\infty}\left(R^{n}\right)$, not identically zero, $\varphi \in \mathscr{C}\left(\mathscr{M}_{1}\right)$. Then $\varphi(x) f(D)$ is not compact if $\varphi$ does not vanish at infinity.

Proof. Using the assumption on $\varphi$, construct a sequence of balls $B_{v}$ $=\left\{x:\left|x-x_{v}\right|<1\right\}$ such that $B_{v} \cap B_{\mu}=\varnothing$ if $\mu \neq \nu$ while $\left|x_{v}\right| \rightarrow \infty$ and $|\varphi(x)| \geqq C>0$ on $\bigcup_{v=1}^{\infty} B_{v}$. If $f(x) \not \equiv 0$ then we can find $u \in L^{2}$ with $f(D) u \neq 0$, and since $f(D)$ commutes with translations we can insure that $\int_{|x| \leqq 1}|f(D) u|^{2} d x>0$. Select a nonzero $w(x) \in C_{0}^{\infty}(|x|<1)$, such that $w(x) f(D) u(x)$ is not identically zero. Define the unitary operator $T_{v}$ on $L^{2}$ by $T_{v} v(x)=v\left(x+x_{v}\right)$, and let $\Omega_{v}=T_{v} w T_{v}^{-1}$. Also set $u_{v}=T_{v} u$. Now

$$
\left\|\Omega_{v} \varphi(x) f(D) u_{v}\right\|=\left\|w(x) \varphi\left(x-x_{v}\right) f(D) u\right\| \geqq C\|w(x) f(D) u\|>0,
$$

using the fact that $|\varphi(x)| \geqq C$ on $B_{v}$. If $v_{v}=\varphi(x) f(D) u_{v}$ had a convergent subsequence, which we continue to write $v_{v}$, say $v_{v} \rightarrow v$, then $\Omega_{v} v_{v} \rightarrow 0$, so a contradiction results. Hence $\varphi(x) f(D) u_{v}$ has no convergent subsequence, while $\left\|u_{v}\right\|=\left\|T_{v} u\right\|=\|u\|$ is bounded. Hence $\varphi(x) f(D)$ cannot be compact.

Let us note in passing that $\varphi \in \mathscr{C}\left(\mathscr{M}_{2}\right)$ implies $\varphi \in \mathscr{C}\left(\mathscr{M}_{1}\right)$.

The proof of the following theorem is also essentially due to H. O. Cordes (unpublished).

THEOREM 1. $\mathscr{M}=\mathscr{M}_{1} \times \mathscr{M}_{2} \mid R_{x}^{n} \times R_{\xi}^{n}$.

Proof. We have identified $\mathscr{M}$ with a subset of $\mathscr{M}_{1} \times \mathscr{M}_{2}$. It remains to see what this subset is. Now clearly if $\left(x_{0}, \xi_{0}\right) \in R_{x}^{n} \times R_{\xi}^{n}$ then $\left(x_{0}, \xi_{0}\right) \notin \mathscr{M}$. For we can take $a \in C_{0}^{\infty}$ with $a\left(x_{0}\right) \neq 0$ and consider $a(x) / Q(\xi)^{1 / 2}$. On the one hand, $a(x) Q^{-1 / 2}$ is compact, and on the other hand $a\left(x_{0}\right) / Q\left(\xi_{0}\right)^{1 / 2} \neq 0$, so $\left(x_{0}, \xi_{0}\right) \notin \mathscr{M}$. It remains to show that every other point of $\mathscr{M}_{1} \times \mathscr{M}_{2}$ does belong to $\mathscr{M}$.

Suppose that $x_{0} \in R_{x}^{n}, \xi_{0} \in \mathscr{M}_{2} \mid R_{\xi}^{n}$, and $\left(x_{0}, \xi_{0}\right) \notin \mathscr{M}$. Since $\mathscr{M}$ is compact, we can find a neighborhood $U$ of $x_{0}$ in $R_{x}^{n}$ and a neighborhood $V$ of $\xi_{0}$ in $\mathscr{M}_{2}$ such that $U \times V \cap \mathscr{M}=\varnothing$. Now we can choose functions $f \in C_{0}^{\infty}(U)$ and $\varphi \in \mathscr{C}\left(\mathscr{M}_{2}\right)$ such that $f\left(x_{0}\right) \neq 0, \varphi\left(\xi_{0}\right) \neq 0$, and supp $\varphi \subset V$. Since $f(x) \varphi(\xi)$ vanishes on $\mathscr{M}$, 
$f(x) \varphi(D)$ must be compact. However, the above lemma implies that $\varphi(x) f^{x}(D)$ is not compact, and hence $\varphi(D) f(x)=F^{-1} \varphi(x) \breve{f}(D) F$ is not compact, so $f(x) \varphi(D)$ cannot be compact. This contradiction shows that $\left(x_{0}, \xi_{0}\right) \in \mathscr{M}$. Similarly $\mathscr{M}$ includes $\left(\mathscr{M}_{1} \backslash R_{x}^{n}\right) \times R_{\xi}^{n}$, and since $\mathscr{M}$ is closed and $R_{x}^{n}$ is dense in $\mathscr{M}_{1}$ and $R_{\xi}^{n}$ dense

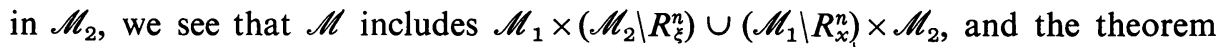
is established.

Note. The above gives an isomorphism $\mathfrak{A} / J \approx \mathscr{C}(\mathscr{M})$, the space of continuous functions on $\mathscr{M}$. The map $\mathfrak{A} \stackrel{\sigma}{\rightarrow} \mathscr{C}(\mathscr{M})$ defines a function $\sigma(A)$ on $\mathscr{M}$, called the symbol of $A \in \mathfrak{A}$. Note that if $a(x)$ is as in (i) then $\sigma(a)=a(x)$, and if $q(D)<P(D)$ then $\sigma\left(q(D) Q^{-1 / 2}\right)=q(\xi) / Q(\xi)^{1 / 2}$, where we assume that $a(x)$ and $q(\xi) / Q(\xi)^{1 / 2}$ have been extended in the natural manner to continuous functions on $\mathscr{M}_{1}$ and $\mathscr{M}_{2}$, respectively.

Theorem 1 provides a necessary and sufficient condition for an element of $\mathfrak{A}$ to be a Fredholm map on $L^{2}\left(R^{n}\right)$, as we shall now see. We neglect the question of whether $\mathfrak{A}$ contains the space of all compact operators $\mathfrak{E}$. As the next proposition shows, this is not important for our purposes. Let $\mathfrak{B}$ be the algebra generated by $\mathfrak{A}$ and $\mathfrak{C}$, and let $\overrightarrow{\mathfrak{B}}$ be its closure, so $\overline{\mathfrak{B}}$ is the $C^{*}$ algebra generated by $\mathfrak{A}$ and $\mathfrak{C}$.

Proposition 3. $\mathfrak{A} / J=\overline{\mathfrak{B}} / \mathfrak{C}$.

Proof. The natural map $\mathfrak{A} \rightarrow \overline{\mathfrak{B}} / \mathfrak{C}$ induced by the inclusion $\mathfrak{A} \rightarrow \overline{\mathfrak{B}}$ has as its kernel $\mathfrak{A} \cap \mathfrak{C}=J$, so we have an injective *-homomorphism $\mathfrak{A} / J \stackrel{\mathfrak{j}}{\rightarrow} \overline{\mathfrak{B}} / \mathfrak{C}$, whose image is easily seen to be $\mathfrak{B} / \mathfrak{C}$, which is dense. But the image of a $C^{*}$ algebra under a *-homomorphism is closed, so $j$ is an isomorphism.

THEOREM 2. An element of $\mathfrak{A}$ is a Fredholm map on $L^{2}\left(R^{n}\right)$ if and only if its symbol on $\mathscr{M}$ is nowhere vanishing.

Proof. $A \in \mathfrak{A}$ is Fredholm if and only if $A$ is invertible in $\mathscr{L}\left(L^{2}\right) / \mathfrak{E}$, where $\mathscr{L}\left(L^{2}\right)$ is the space of bounded operators on $L^{2}$, which is true if and only if $A$ is invertible in $\overline{\mathfrak{B}} / \mathfrak{C}$, since $\overline{\mathfrak{B}}$ is a $C^{*}$ algebra containing $A$. By Proposition 3 , this is equivalent to $A$ being invertible in $\mathfrak{A} / J$, which of course is true if and only if the symbol of $A$ on $\mathscr{M}$ is nowhere vanishing.

If $\left\{q_{j}(\xi): j=1, \ldots, K\right\}$ is a basis of the finite-dimensional space of polynomials weaker than $P(\xi)$, it is easy to see that the differential operators of the form $\sum a_{j}(x) q_{j}(D)$ with $a_{j}$ functions as in (i) are precisely the differential operators $p(x, D)$ whose coefficients belong to $C^{\infty}\left(R^{n}\right) \cap L^{\infty}\left(R^{n}\right)$ and have all nonzero order derivatives vanishing at infinity, such that $p\left(x_{0}, D\right)<P(D)$ for each $x_{0} \in R^{n}$. All such operators of course have the property that $p(x, D) Q^{-1 / 2} \in \mathfrak{U}$, so we have a criterion on which $p(x, D)$ is a Fredhom map from $\mathfrak{B}_{2, \tilde{P}}$ to $L^{2}$.

THEOREM 3. Let $p(x, D)$ be a differential operator on $R^{n}$ whose coefficients belong to $C^{\infty}\left(R^{n}\right) \cap L^{\infty}\left(R^{n}\right)$ and have all nonzero order derivatives vanishing at infinity. Suppose that $p\left(x_{0}, D\right)<P(D)$ for each $x_{0} \in R^{n}$, where $P(D)$ is a hypoelliptic operator. Then the following form a necessary and sufficient condition that $p(x, D) Q^{-1 / 2}$ be a 
Fredholm map on $L^{2}\left(R^{n}\right)$, or equivalently, that $p(x, D): \mathfrak{B}_{2, \tilde{P}}\left(R^{n}\right) \rightarrow L^{2}\left(R^{n}\right)$ be a Fredholm map.

(a) There is an $R<\infty$ and a $\delta>0$ such that $|p(x, \xi)| / Q(\xi)^{1 / 2} \geqq \delta$ for all $\xi$ such that $|\xi| \geqq R$, and for all $x \in R^{n}$, or equivalently we may require that $|p(x, \xi) / P(\xi)| \geqq \delta$ for all $|\xi| \geqq R, x \in R^{n}$. (This inequality expresses that $p(x, D)$ has the same strength as $P(D)$, uniformly on $R^{n}$.)

(b) There is an $R<\infty$ and a $\delta>0$ such that $|p(x, \xi)| \geqq \delta$ for all $\xi \in R^{n}$ and all $x \in R^{n}$ such that $|x| \geqq R$.

Under the hypotheses given on $p(x, D),(\mathrm{a})$ and (b) above are also necessary and sufficient in order that $p(x, D): \mathfrak{B}_{2, \tilde{F} k} \rightarrow \mathfrak{B}_{2, k}$ be a Fredholm map, where $k(\xi)$ $=\left(1+\left|P_{1}(\xi)\right|^{2}\right)^{t}$, with $P_{1}(D)$ another hypoelliptic operator with constant coefficients and $t$ any real number.

Proof. Everything but the last statement is an immediate consequence of Theorem 2, together with the fact that the symbol of $p(x, D) Q^{-1 / 2}$ is $p(x, \xi) / Q(\xi)^{1 / 2}$. The last statement follows immediately from the next proposition.

Proposition 4. Let $A \in \mathfrak{A}_{0}$, the algebra (not closed) generated by the operators given in (i) and (ii) at the first of this section, and suppose $\Lambda=I+P_{1}(D)^{t} P_{1}(D)$, where $P_{1}(D)$ is any hypoelliptic operator. Then for any $t \in R, \Lambda^{t} A \Lambda^{-t}-A$ is a compact operator on $L^{2}$. Hence $\Lambda^{t} A \Lambda^{-t} \in \overline{\mathfrak{B}}$ and has the same symbol as $A$, which is a function on the maximal ideal space of $\overline{\mathfrak{B}} / \mathfrak{S}=\mathfrak{Q} / J$.

Proof. It suffices to show that $\Lambda^{t} A \Lambda^{-t}-A$ is compact when $A$ is a generator of $\mathfrak{A}_{0}$, and since $\Lambda^{t} A \Lambda^{-t}-A=0$ if $A=f(D)$, it suffices to consider $\Lambda^{t} a(x) \Lambda^{-t}-a(x)$ with $a(x)$ as in (i). Now Proposition 1 says that there is a compact operator $C$ such that $a(x) \Lambda^{-t}-\Lambda^{-t} a(x)=C \Lambda^{-t}$ when $t>0$. Hence $a(x)-\Lambda^{-t} a(x) \Lambda^{t}=C$, so $\Lambda^{-t} a(x) \Lambda^{t}-a(x)$ is compact when $t>0$. On the other hand, taking adjoints and replacing $a(x)$ by $\tilde{a}(x)$, we have $\Lambda^{-t} a(x)-a(x) \Lambda^{-t}=\Lambda^{-t} C^{*}$, and $C^{*}$ is compact. Hence $a(x)-\Lambda^{t} a(x) \Lambda^{-t}=C^{*}$, and so $\Lambda^{t} a(x) \Lambda^{-t}-a(x)$ is compact, for $t>0$. This completes the proof.

II. Spectral theory and regularity theorems. Let $P(D)$ be hypoelliptic, $Q=I+P(D)^{t} P(D)$. Suppose $p(x, D)$ is an operator whose coefficients belong to $C^{\infty}\left(R^{n}\right) \cap L^{\infty}\left(R^{n}\right)$ and all of whose nonzero order derivatives vanish at infinity, such that $p\left(x_{0}, D\right)<P(D)$ for each $x_{0} \in R^{n}$. Suppose $p(x, D)$ is formally selfadjoint, i.e., $(p(x, D) \varphi, \psi)=(\varphi, p(x, D) \psi)$ if $\varphi, \psi \in C_{0}^{\infty}\left(R^{n}\right)$ (and hence if $\varphi, \psi \in \mathfrak{B}_{2, \tilde{P}}$ ). It follows that $Q^{-1 / 4} p(x, D) Q^{-1 / 4}$ is a selfadjoint operator on $L^{2}\left(R^{n}\right)$. Hence its symbol $\sigma\left(Q^{-1 / 4} p(x, D) Q^{-1 / 4}\right)=\sigma\left(p(x, D) Q^{-1 / 2}\right)$ is real valued on $\mathscr{M}$. We now assume that $p(x, D)$ is uniformly equally as strong as $P(D)$ on $R^{n}$, i.e., there are constants $R<\infty$ and $\delta>0$ such that $|p(x, \xi) / P(\xi)| \geqq \delta$ when $|\xi| \geqq R$, for all $x \in R^{n}$, or equivalently, that $\sigma\left(p(x, D) Q^{-1 / 2}\right)$ does not vanish on $S$, where $S$ $=\mathscr{M}_{1} \times\left(\mathscr{M}_{2} \mid R^{n}\right)$. If $n \geqq 2$, it follows that $p(x, \xi)$ cannot change sign, so is always positive or always negative, when $|\xi| \geqq R$. We shall assume it is positive, so that 
$\sigma\left(p(x, D) Q^{-1 / 2}\right) \geqq \delta>0$ on $S$. Hence $\sigma\left(p(x, D) Q^{-1 / 2}\right)>0$ on a neighborhood of $S$. From this it is easy to see that if $\lambda \neq 0$ then $\sigma\left((p(x, D)+i \lambda) Q^{-1 / 2}\right)$ is nonvanishing on $\mathscr{M}$ and homotopic to $1=\sigma(I)$ through nonvanishing symbols. It follows that $p(x, D)+i \lambda$ is a Fredholm map of $\mathfrak{B}_{2, \tilde{P}} \rightarrow L^{2}$, and, by Theorem 4 of Breuer and Cordes [1] (Theorem 7 of [2]), its index is zero. Similarly, if $\lambda>0$ is sufficiently large, then $p(x, D)+\lambda$ is a Fredholm map of $\mathfrak{B}_{2, \tilde{P}} \rightarrow L^{2}$ and its index is zero. Note that the same facts hold for $Q^{-t} p(x, D) Q^{t}+i \lambda$ and $Q^{-t} p(x, D) Q^{t}+\lambda$.

In the rest of this chapter, $p(x, D)$ will be as above, $P_{0}$ will be the operator $p(x, D)$ with domain $\mathscr{D}\left(P_{0}\right)=H^{\infty}\left(R^{n}\right)$, and $P$ will be the operator $p(x, D)$ with domain $\mathscr{D}(P)=\mathfrak{B}_{2, \tilde{P}}$. $(P$ is not to be confused with the constant coefficients operator $P(D)$.) We shall also set $P^{\prime}=Q^{-t} p(x, D) Q^{t}$ with domain $\mathfrak{B}_{2, \tilde{P}}$.

\section{THEOREM 1. $P$ and $P^{\prime}$ are selfadjoint.}

Proof. As is well known, $P$ is selfadjoint if $P+i \lambda$ is onto when $\lambda \neq 0$. But we have seen that $P+i \lambda$ is a Fredholm operator with index zero. On the other hand, $\operatorname{Im}((P+i \lambda) u, u)=\lambda\|u\|^{2}$ if $u \in \mathfrak{B}_{2, \tilde{P}}$, so $P+i \lambda$ is injective. Hence it is surjective. The same argument applies to $P^{\prime}$.

Note that, since $P_{0} \subset P \subset \bar{P}_{0}, P_{0}$ is essentially selfadjoint.

Corollary. If $u \in L^{2}$ and $p(x, D) u \in L^{2}$, then $u \in \mathfrak{B}_{2, \tilde{P}}$.

Proof. The hypothesis implies that $u \in \mathscr{D}\left(P_{0}^{*}\right)$. In fact, if $\varphi \in H^{\infty}$, then $(u, p(x, D) \varphi)$ $=(p(x, D) u, \varphi)$ since $p(x, D)$ is formally selfadjoint. But since $P_{0} \subset P \subset \bar{P}_{0}$, we have $P_{0}^{*}=P^{*}=P$, so $u \in \mathscr{D}(P)=\mathfrak{B}_{2, \tilde{P}}$.

Note that, similarly, if $u \in L^{2}$ and $Q^{-t} p(x, D) Q^{t} u \in L^{2}$, then $u \in \mathfrak{B}_{2, \tilde{P}}$.

Let now $q(x, D)$ be another operator which is weaker than $P(D)$ and whose coefficients satisfy the same conditions as given above for the coefficients of $p(x, D)$. We assume $q(x, D)$ has the same strength as $P(D)$, uniformly on $R^{n}$, i.e., there are constants $R<\infty$ and $\delta>0$ such that $|q(x, \xi) / P(\xi)| \geqq \delta$ if $|\xi| \geqq R, x \in R^{n}$. or equivalently, $\sigma\left(q(x, D) Q^{-1 / 2}\right)$ is nonvanishing on $S$. It follows that $\sigma\left(q(x, D)^{t} q(x, D) Q^{-1}\right)$ is strictly positive on $S$. Also, $q(x, D)^{t} q(x, D)$ is formally selfadjoint, where $q(x, D)^{t}$ is the formal adjoint of $q(x, D)$. Thus we can apply the above corollary (with $P(D)$ replaced by $\left.P(D)^{2}\right)$ to $q(x, D)^{t} q(x, D)$ to conclude that $q(x, D)^{t} q(x, D) u \in L^{2}, \quad u \in L^{2}$ implies $u \in \mathfrak{B}_{2, \tilde{P}^{2}}$. Hence $q(x, D) u \in \mathfrak{B}_{2, \tilde{P}}, \quad u \in L^{2}$ implies $u \in \mathfrak{B}_{2, \tilde{P}^{2}}$. The open mapping theorem applied to the identity map $j: \mathfrak{B}_{2, \tilde{P}^{2}}$ $\rightarrow F$ where $F=\left\{u \in L^{2}: q(x, D) u \in \mathfrak{B}_{2, \not}\right\}$ implies, since $j$ is a bijective continuous mapping between Banach spaces, that $\|u\|_{\mathcal{P}^{2}} \leqq C\|q(x, D) u\|_{\tilde{P}}+C\|u\|$ for all $u \in \mathfrak{B}_{2, \tilde{F}^{2}}$ for some constant $C$ independent of $u$. Replacing $u$ by $Q^{-1 / 2} v, v \in \mathfrak{B}_{2, \text { }}$, we see that $\|v\|_{\tilde{P}} \leqq C\left\|Q^{1 / 2} q(x, D) Q^{-1 / 2} v\right\|+C\|v\|_{1 / \tilde{P}}$ for all $v \in \mathfrak{B}_{2, \tilde{P}}$. Now all the above is valid if $q(x, D)$ is replaced by $Q^{-1 / 2} q(x, D) Q^{1 / 2}$, so we conclude that $\|v\|_{\tilde{P}} \leqq$ $C\|q(x, D) v\|+C\|v\|_{1 / \tilde{P}} \leqq C\|q(x, D) v\|+C\|v\|$. We can restate this as follows.

THEOREM 2. If $q(x, D)$ is as above, and if $T=q(x, D)$ with domain $\mathscr{D}(T)=\mathfrak{B}_{2, \tilde{P}}$ then $T$ is closed. 
To prove our next theorem, we make use of the following lemma, proved in Nelson and Stinespring [8].

Lemma. Let $\mathscr{D}$ be a dense linear mainfold in a Hilbert space $H$. Let $T_{0}$ and $W_{0}$ be linear transformations whose domains are $\mathscr{D}$ and whose ranges are contained in $\mathscr{D}$ such that $T_{0}$ is contained in the adjoint of $W_{0}$. If $T_{0} W_{0}$ is essentially selfadjoint, then $\bar{T}_{0}=W_{0}^{*}$.

Now we take $T_{0}=q(x, D)$ with $\mathscr{D}\left(T_{0}\right)=H^{\infty}\left(R^{n}\right), W_{0}=q(x, D)^{t}$ with $\mathscr{D}\left(W_{0}\right)$ $=H^{\infty}\left(R^{n}\right)$, and $W=q(x, D)^{t}$ with $\mathscr{D}(W)=\mathfrak{B}_{2, \text { ₹ }}$.

THEOREM 3. $T=W^{*}$.

Proof. We see that the hypotheses of the above lemma are fulfilled by these operators, with $\mathscr{D}=H^{\infty}\left(R^{n}\right)$. Hence $W_{0}^{*}=T_{0}$. Since $T_{0} \subset T \subset \bar{T}_{0}$ and, by Theorem 2, $T$ is closed, we have $T=\bar{T}_{0}$. Since $W_{0} \subset W \subset \bar{W}_{0}$, we have $W_{0}^{*}=W^{*}$. The proof is complete.

This gives us a regularity theorem, extending the corollary to Theorem 1 .

TheOREM 4. If $u \in L^{2}$ and $q(x, D) u \in L^{2}$, then $u \in \mathfrak{B}_{2, \tilde{p}}$; if $q(x, D) u \in H^{\infty}, u \in H^{\infty}$.

Proof. The hypothesis guarantees that $u \in \mathscr{D}\left(W_{0}^{*}\right)$ since $\varphi \in H^{\infty}$ implies $\left(u, q(x, D)^{t} \varphi\right)=(q(x, D) u, \varphi)$. But by Theorem $3, W_{0}^{*}=W^{*}=T$, so $u \in \mathscr{D}(T)=\mathfrak{B}_{2, \tilde{p}}$. The second assertion follows if $q(x, D)$ is replaced by $P(D)^{j} q(x, D)$ and we let $j \rightarrow \infty$.

We return now to the operator $p(x, D)$, which, we recall, is formally selfadjoint and satisfies the condition $\sigma\left(Q^{-1 / 4} p(x, D) Q^{-1 / 4}\right)>0$ on $S$. Hence, for $\lambda>0$ sufficiently large, $\sigma\left(Q^{-1 / 4}(p(x, D)+\lambda) Q^{-1 / 4}\right)>0$ on $\mathscr{M}$. If we let

$$
A=Q^{-1 / 4}(p(x, D)+\lambda) Q^{-1 / 4},
$$

then $A$ is a bounded selfadjoint operator on $L^{2}$, and the condition $\sigma(A)>0$ on $\mathscr{M}$ implies that there is a positive operator $A_{0} \geqq \eta I$ (for some $\eta>0$ ) and a compact operator $K$ such that $A=A_{0}-K$. This implies that the continuous spectrum of $A$ lies in $[\eta, \infty)$, and that all eigenvalues less than $\eta$ correspond to eigenspaces of finite dimension. Thus we can take $K$ to be given by $K u=\sum_{j=1}^{v} \lambda_{j}\left(u, u_{j}\right) u_{j}$ where $\left\{-\lambda_{j}: 1 \leqq i \leqq \nu\right\}$ are the negative eigenvalues of $A$ (counted with multiplicities) and $u_{j}$ corresponding eigenvectors. $\left(A+\lambda_{j}\right) u_{j}=0$. Note that $A+\lambda_{j}=Q^{-1 / 4}\left(p(x, D)+\lambda_{j} Q^{1 / 2}+\lambda\right) Q^{-1 / 4}$. Now, as indicated in several previous remarks, all the reasoning in Theorem 4 and its corollary applies to the operator $Q^{1 / 4}\left(p(x, D)+\lambda_{j} Q^{1 / 2}+\lambda\right) Q^{-1 / 4}$. Hence we conclude that each $u_{j} \in H^{\infty}$. Now we have, for $u \in \mathfrak{B}_{2, \tilde{\beta}},((p(x, D)+\lambda) u, u)=\left(A Q^{1 / 4} u\right.$, $\left.Q^{1 / 4} u\right)=\left(A_{0} Q^{1 / 4} u, Q^{1 / 4} u\right)-\left(K Q^{1 / 4} u, Q^{1 / 4} u\right) \geqq \eta\left\|Q^{1 / 4} u\right\|^{2}-\left\|K^{1 / 2} Q^{1 / 4} u\right\|^{2}$. But

$$
K^{1 / 2} Q^{1 / 4} u=\sum_{j=1}^{v} \sqrt{ } \lambda_{j}\left(Q^{1 / 4} u, u_{j}\right) u_{j}=\sum_{j=1}^{v} \sqrt{ } \lambda_{j}\left(u, Q^{1 / 4} u_{j}\right) u_{j}
$$

so since $Q^{1 / 4} u_{j} \in L^{2}$ we have $\left\|K^{1 / 2} Q^{1 / 4} u\right\|^{2} \leqq C^{\prime}\|u\|^{2}$. Hence $((p(x, D)+\lambda) u, u$ $\geqq C\|u\|_{\rho^{1 / 2}}^{2}-C^{\prime}\|u\|^{2}$ so with $\lambda^{\prime}=\lambda+C^{\prime}$, we have the following inequality:

$$
\left(\left(p(x, D)+\lambda^{\prime}\right) u, u\right) \geqq C\|u\|_{P^{1 / 2}}^{2} \text {. }
$$

In particular, we see that $P$ is semibounded. 
We can apply the previous reasoning to an operator $r(x, D)$, whose coefficients are smooth and satisfy the same restrictions at infinity as the other operators considered in this chapter, which is uniformly strongly formally hypoelliptic, i.e. which satisfies $\operatorname{Re} r(x, \xi) / Q(\xi)^{1 / 2} \geqq \delta>0$ when $|\xi| \geqq R$, for all $x \in R^{n}$, or equivalently $\operatorname{Re} \sigma\left(r(x, D) Q^{-1 / 2}\right)>0$ on $S$. Again note that for $\lambda>0$ sufficiently large, $\operatorname{Re} \sigma\left((r(x, D)+\lambda) Q^{-1 / 2}\right)>0$ on $\mathscr{M}$, so $r(x, D)+\lambda: \mathfrak{B}_{2, \tilde{P}} \rightarrow L^{2}$ is a Fredholm map of index zero. Note that $p(x, D)=\frac{1}{2} r(x, D)+\frac{1}{2} r(x, D)^{t}$ satisfies the conditions given above for $p(x, D)$. Hence we have the following Gårding type inequality. For $u \in C_{0}^{\infty}(K)$ where $K$ is a fixed compact set in $R^{n}$, this inequality was established in Malgrànge [7].

TheOREM 5. $\operatorname{Re}(r(x, D) u, u) \geqq C\|u\|_{\widetilde{P}^{1 / 2}}^{2}-C^{\prime}\|u\|^{2}$ for all $u \in \mathfrak{B}_{2, \tilde{P}}$, where $C$ and $C^{\prime}$ are constants independent of $u$.

Proof. $\operatorname{Re}(r(x, D) u, u)=(p(x, D) u, u)$, so the theorem follows from the inequality $(*)$ above.

This theorem implies in particular that, if $\lambda>0$ is sufficiently large, then $\operatorname{Re}((r(x, D)+\lambda) u, u) \geqq C\|u\|_{\widetilde{P}^{1 / 2}}^{2}>0$, so $r(x, D)+\lambda$ is accretive, and also injective. But since, for $\lambda>0$ large, $r(x, D)+\lambda$ is a Fredholm operator of index zero, we see that $r(x, D)+\lambda: \mathfrak{B}_{2, \tilde{P}} \rightarrow L^{2}$ is a bijective map, so $-\lambda$ belongs to the resolvent set of $r(x, D)$. This implies that $r(x, D)+\lambda$ is a maximal accretive operator, and so $-r(x, D)-\lambda$ generates a contraction semigroup. Hence, $-r(x, D)$, with domain $\mathfrak{B}_{2, \tilde{P}}$, generates a strongly continuous semigroup.

In concluding this section, let us note that from Theorem 4 we can deduce the local regularity theorem for operators of constant strength $q(x, D)$ which are formally hypoelliptic. First, there is no restriction in assuming the coefficients of $q(x, D)$ are altered in a neighborhood of infinity so that $q(x, D)$ satisfies all the conditions imposed on the operator in Theorem 4 . Note also that Theorem 4 can

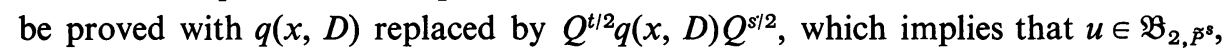
$q(x, D) u \in \mathfrak{B}_{2, \tilde{P}^{t}} \Rightarrow u \in \mathfrak{B}_{2, \tilde{P}^{(1+t)}}$.

Now suppose that $u \in \mathscr{D}^{\prime}(\Omega), q(x, D) u \in C^{\infty}(\Omega)$. For every relatively compact $\Omega_{0} \subset \Omega$, we have $u \in \mathfrak{B}_{2, \tilde{P}}^{\text {loc }} s\left(\Omega_{0}\right)$ for some real number $s$. Thus $\varphi u \in \mathfrak{B}_{2, \tilde{P}^{s}}\left(R^{n}\right)$ if $\varphi \in C_{0}^{\infty}\left(\Omega_{0}\right)$. If $q(x, D)=\sum_{j=1}^{k} a_{j}(x) q_{j}(D)$, with $a_{j}(x)$ functions belonging to $C^{\infty}\left(R^{n}\right) \cap L^{\infty}\left(R^{n}\right)$ whose nonzero order derivatives vanish at infinity, and $q_{j}(D)<P(D)$, then

$$
q(x, D) \varphi u=\varphi q(x, D) u+\sum_{j=1}^{k} \sum_{\alpha \neq 0} \frac{1}{\alpha !} a_{j}(x) D^{\alpha} \varphi(x) q_{j}^{(\alpha)}(D) u .
$$

The first term belongs to $C_{0}^{\infty}\left(R^{n}\right)$, by assumption. From Lemma 1 of $\S \mathrm{I}$ it follows that the second term, and hence $q(x, D) \varphi u$, belongs to $\mathfrak{B}_{2, \tilde{P}^{s-1+\nu}}\left(R^{n}\right)$ where $\gamma=\rho / \nu$, with $\rho$ the constant used in that lemma and $\nu$ the order of $P(\xi)$. Thus $\varphi u \in \mathfrak{B}_{2, \tilde{P}^{s+\gamma}}$, so $u \in \mathfrak{B}_{2, \tilde{P}^{s+\gamma}}^{\text {loc }}\left(\Omega_{0}\right)$. Applying this reasoning repeatedly, we get $u \in \mathfrak{B}_{\tilde{P}^{s}+j \gamma}^{\text {loc }}\left(\Omega_{0}\right)$, and letting $j \rightarrow \infty$, we get $u \in C^{\infty}\left(\Omega_{0}\right)$. Hence $u \in C^{\infty}(\Omega)$. 
III. An algebra of pseudo differential operators. In order to exhibit the theorems about differential operators proved in $\S I I$, together with their generalizations to other operators used from time to time in that section, in a unified framework, we shall define an algebra of pseudo differential operators for which theorems analogous to those in $\S \mathrm{II}$ can be proved.

Definition 1. A continuous operator $A$ on $L^{2}\left(R^{n}\right)$ belongs to $P S(0)$ if and only if $A \in \overline{\mathfrak{B}}$ and for all $t \in R, Q^{t} A Q^{-t} \in \overline{\mathfrak{B}}$ (i.e. extends or restricts to a continuous operator on $L^{2}$ which belongs to $\overline{\mathfrak{B}}$ ) and $Q^{t} A Q^{-t}-A$ is compact.

As we have seen in Proposition 4 of $\S I$, every element of $\mathfrak{A}_{0}$ belongs to $P S(0)$. Also if $A \in \mathfrak{A}_{0}$ and $\Lambda$ is as in that proposition, $\Lambda^{s} A \Lambda^{-s} \in P S(0)$ for all real $s$.

Proposition 1. PS(0) is an algebra.

Proof. Obviously if $A_{1}, A_{2} \in P S(0), A_{1}+A_{2} \in P S(0)$. Also, if $s \in R$, then $Q^{s} A_{1} A_{2} Q^{-s}=Q^{s} A_{1} Q^{-s} Q^{s} A_{2} Q^{-s}=\left(A_{1}+K_{1}\right)\left(A_{2}+K_{2}\right)$ where $K_{1}$ and $K_{2}$ are compact, so the assertion follows.

In the remainder of this section, let $H^{r}\left(R^{n}\right)=\mathfrak{B}_{2, \tilde{P}^{r}}\left(R^{n}\right)$.

Definition 2. A continuous operator $A: H^{r}\left(R^{n}\right) \rightarrow L^{2}\left(R^{n}\right)$ belongs to $P S(r), r$ a given real number, if $A Q^{-r / 2} \in P S(0)$. In such a case, we define the $r$-symbol of $A$ to be $\sigma_{r}(A)=\sigma\left(A Q^{-r / 2}\right)$.

Note that if $A \in P S(r)$, then $A: H^{s}\left(R^{n}\right) \rightarrow H^{s-r}\left(R^{n}\right)$ continuously, for all real $s$ (after extension or restriction).

Proposition 2. PS( $r)$ is a linear space. If $A \in P S(r)$, then $Q^{s} A Q^{-s} \in P S(r)$ for all real $s$, and $\sigma_{r}\left(Q^{s} A Q^{-s}\right)=\sigma_{r}(A)$. If $B \in P S\left(r^{\prime}\right)$, then $A B \in P S\left(r+r^{\prime}\right)$ and $\sigma_{r+r^{\prime}}(A B)$ $=\sigma_{r}(A) \sigma_{r}(B)$.

Proof. The first assertion is trivial. As for the second, we are given that $A Q^{-r / 2} \in P S(0)$ and are to show that $Q^{s} A Q^{-s-r / 2} \in P S(0)$. Now if $t$ is a real number, $Q^{t}\left(Q^{s} A Q^{-s-r / 2}\right) Q^{-t}=Q^{s+t}\left(A Q^{-r / 2}\right) Q^{-s-t}=A Q^{-r / 2}+K_{1}$ with $K_{1}$ compact, since $A Q^{-r / 2} \in P S(0)$. But also $Q^{s} A Q^{-s-r / 2}=Q^{s}\left(A Q^{-r / 2}\right) Q^{-s}=A Q^{-r / 2}+K_{2}$ with $K_{2}$ compact for the same reason, so $Q^{t}\left(Q^{s} A Q^{-s-r / 2}\right) Q^{-t}=Q^{s} A Q^{-s-r / 2}+K_{1}-K_{2}$. This establishes the second assertion, and also the identity of the symbols. We must now show that $A B Q^{-r / 2-r^{\prime} / 2} \in P S(0)$. By the result just proved, it suffices to show that $Q^{-r / 2} A B Q^{-r^{\prime} / 2} \in P S(0)$. But $B Q^{-r^{\prime} / 2} \in P S(0)$ by assumption, and $Q^{-r / 2} A=Q^{-r / 2}\left(A Q^{-r / 2}\right) Q^{r / 2} \in P S(0)$, again by the assertion just proved, so $Q^{-r / 2} A B Q^{-r^{\prime} / 2} \in P S(0)$ because $P S(0)$ is an algebra. Finally, $\sigma_{r+r^{\prime}}(A B)=$ $\sigma\left(A B Q^{-r / 2-r^{\prime} / 2}\right)=\sigma\left(Q^{-r / 2} A B Q^{-r^{\prime} / 2}\right)=\sigma\left(Q^{-r / 2} A\right) \sigma\left(B Q^{-r^{\prime} / 2}\right)=\sigma_{r}(A) \sigma_{r^{\prime}}(B)$.

Since if $A \in P S(r), A: H^{s} \rightarrow H^{s-r}$ continuously for each real $s$, if ( , ) denotes the dual pairing between $H^{t}$ and $H^{-t}$, linear in the first argument and conjugate linear in the second, we see that there is a continuous mapping $A^{t}: H^{-s+r} \rightarrow H^{-s}$, called the formal adjoint of $A$, such that $(A u, v)=\left(u, A^{t} v\right)$ for $u \in H^{s}, v \in H^{-s+r}$.

Proposition 3. If $A \in P S(r)$, then $A^{t} \in P S(r) . \sigma_{r}\left(A^{t}\right)=\bar{\sigma}_{r}(A)$. 
Proof. To show that $A^{t} Q^{-r / 2} \in P S(0)$, it suffices to show that $Q^{-r / 2} A^{t} \in P S(0)$. Now $Q^{s}\left(A Q^{-r / 2}\right) Q^{-s}-A Q^{-r / 2}=K$ where $K$ is a compact operator on $L^{2}$. Taking adjoints, we get $Q^{-s}\left(Q^{-r / 2} A^{t}\right) Q^{s}-Q^{-r / 2} A=K^{*}$, and $K^{*}$ is compact, so the first assertion is established. The second assertion is true because $\sigma_{r}\left(A^{t}\right)=\sigma\left(Q^{-r / 4} A^{t} Q^{-r / 4}\right)$ $=\sigma\left(\left(Q^{-r / 4} A Q^{-r / 4}\right)^{*}\right)=\bar{\sigma}_{r}(A)$.

We are now in a position to state the analogues to the theorems of §II. The proofs are identical and will not be repeated. We shall let $P \in P S(r)$ be a formally selfadjoint operator, i.e. $P=P^{t}$, such that $\sigma_{r}(P)>0$ on $S$. We shall let $T \in P S(r)$ be an operator such that $\sigma_{r}(T) \neq 0$ on $S$. In the first theorem we shall assume $r>0$ and consider $P, T$, and $T$ to be unbounded operators on $L^{2}\left(R^{n}\right)$ with domain $\mathscr{D}(P)$ $=\mathscr{D}(T)=\mathscr{D}\left(T^{t}\right)=H^{r}$.

THEOREM 1. $P$ is selfadjoint. More generally, $T$ is closed and $T^{*}=T^{t}$. If $u \in L^{2}$ and $T u \in L^{2}$ (here we consider $T: L^{2} \rightarrow H^{-r}$ ) then $u \in H^{r}$.

THEOREM 2. If $u \in H^{t}$ and $T u \in H^{s}$, then $u \in H^{s+r}$.

Proof. Apply above with $T$ replaced by $Q^{s / 2} T Q^{t / 2}$.

THEOREM 3. If $\operatorname{Re} \sigma_{r}(T)>0$ on $S$, then for $\lambda>0$ sufficiently large, $-T-\lambda$ generates a strongly continuous contraction semigroup of operators on $L^{2}$.

We turn now to the problem of constructing a parametrix for an element of $P S(r)$ whose $r$-symbol does not vanish on $S$.

THEOREM 4. If $T \in P S(r)$ and $\sigma_{r}(T) \neq 0$ on $\mathscr{M}$, there is a $V \in P S(-r)$ such that $V T=I+K_{1}$ and $T V=I+K_{2}$ where $K_{1}$ and $K_{2}$ are continuous mappings of $H^{-\infty} \rightarrow H^{\infty}$ with finite-dimensional range.

Proof. Consider $T: H^{-\infty} \rightarrow H^{-\infty}$. We know that $\operatorname{ker} T=F_{1}$ is a finite-dimensional space consisting of elements of $H^{\infty}$. Also $F_{2}=$ range of $T$ is closed and has finite codimension, and $F_{3}=\operatorname{ker} T^{t}$ is a finite-dimensional space consisting of elements of $H^{\infty}$, supplementary to $F_{2}$. We define $V: H^{-\infty} \rightarrow H^{-\infty}$ as follows. Let $V u=0$ if $u \in F_{3}$, and if $u \in F_{2}$ let $V u=v$ where $v$ is the unique element of $H^{-\infty}$ such that $T v=u$ and $(w, v)=0$ for all $w \in \operatorname{ker} T$. Then $V: H^{s} \rightarrow H^{s-r}$ by the regularity theorem, and this map is continuous, by the closed graph theorem. Note that $I-V T$ is a continuous projection on $F_{1}$ and $I-T V$ is a continuous projection on $F_{3}$. It remains only to show that $V \in P S(-r)$, i.e. $V Q^{r / 2} \in P S(0)$. Since $\sigma_{r}(T) \neq 0$ on $\mathscr{M}$, there is an $A \in \overline{\mathfrak{B}}$ such that $A B-I$ and $B A-I$ are compact, whenever $B \in \overline{\mathfrak{B}}$ is such that $\sigma(B)=\sigma_{r}(T)$. In particular, $Q^{-s} Q^{-r / 2} T Q^{s} A-I=K$, compact. Note that $Q^{-s} V Q^{(r+s) / 2}: L^{2} \rightarrow L^{2}$ continuously, for each real $s$. Hence

$$
\begin{aligned}
K^{\prime} & =Q^{-s} V Q^{(r+s) / 2} K=Q^{-s} V Q^{(r+s) / 2}\left(Q^{-s-r / 2} T Q^{s} A-I\right) \\
& =Q^{-s} V T Q^{s} A-Q^{-s}\left(V Q^{r / 2}\right) Q^{s}=Q^{-s}\left(I+K_{1}\right) Q^{s} A-Q^{-s}\left(V Q^{r / 2}\right) Q^{s} \\
& =A-Q^{-s}\left(V Q^{r / 2}\right) Q^{s}+K^{\prime \prime},
\end{aligned}
$$

so $A-Q^{-s}\left(V Q^{r / 2}\right) Q^{s}$ is compact, for all $s$, which implies that $V Q^{r / 2} \in P S(0)$. 
LEMMA. If $P \in P S(r)$ and if $\sigma_{r}(P)>0$ on $S$, there is an $E \in P S(-r)$ such that $E P-I$ and $P E-I$ are members of $P S(-\infty)=\bigcap_{t>0} P S(-t)$.

Proof. We are given that $\sigma_{r}(P)>0$ on $S$, hence on a neighborhood of $S$. Hence there is a continuous function $f$ on $\mathscr{M}$ which is equal to $\sigma_{r}(P)$ on a neighborhood of $S$ and which is $>0$ on $\mathscr{M}$, and we can find a finite sum $\sum_{j=1}^{N} a_{j}(x) \varphi_{j}(\xi)$ where $a_{j}$ are functions as in (i) of $\S \mathrm{I}$ and $\varphi_{j} \in C_{0}^{\infty}\left(R^{n}\right)$, such that $\sum a_{j}(x) \varphi_{j}(\xi)$ approximates $f-\sigma_{r}(P)$ arbitrarily closely on $\mathscr{M}$. Thus we can assume that $\sigma_{r}\left(P+\sum a_{j}(x) \varphi_{j}(D) Q^{r / 2}\right)$ $>0$ on $\mathscr{M}$. Note that $K_{0}=\sum a_{j}(x) \varphi_{j}(D) Q^{r / 2} \in P S(-\infty)$. Let $P^{\prime}=P+K_{0}$. By Theorem 4, there is an $E \in P S(-r)$ such that $P^{\prime} E-I$ and $E P^{\prime}-I$ belong to $P S(-\infty)$. Hence $P E-I=P^{\prime} E-I+K_{0} E \in P S(-\infty)$ and similarly $E P-I \in P S(-\infty)$.

TheOREM 5. If $T \in P S(r)$ and $\sigma_{r}(T) \neq 0$ on $S$, there is a $V \in P S(-r)$ such that $V T-I$ and $T V-I$ belong to PS $(-\infty)$.

Proof. $P=T^{t} T$ is an element of $P S(2 r)$ such that $\sigma_{2 r}(P)>0$ on $S$. By the above lemma, there is an $E \in P S(-2 r)$ such that $E P-I$ and $P E-I$ belong to $P S(-\infty)$. Let $V=E T^{t}$. Then $V \in P S(-r)$ and $V T-I=E T^{t} T-I=E P-I \in P S(-\infty)$. Let $E^{\prime} \in P S(-2 r)$ be such an inverse $\bmod P S(-\infty)$ of $P^{\prime}=T T^{t}$, and let $V^{\prime}=T^{t} E^{\prime}$. Then $T V^{\prime}-I=T T^{t} E^{\prime}-I=P^{\prime} E^{\prime}-I \in P S(-\infty)$. But evaluating $V T V^{\prime}$ in two ways, we have $V T V^{\prime}=\left(I+L_{1}\right) V^{\prime}=V^{\prime}+L_{1} V^{\prime}$ and $V T V^{\prime}=V\left(I+L_{2}\right)=V+V L_{2}$, so $V^{\prime}-V=V L_{2}$ $-L_{1} V^{\prime} \in P S(-\infty)$. Hence $T V-I \in P S(-\infty)$.

It is of interest to consider a smaller class of pseudo differential operators, whose elements have kernels which are $C^{\infty}$ off the diagonal.

Definition. A continuous linear operator $T: H^{-\infty} \rightarrow H^{-\infty}$ belongs to $O P(r)$ if and only if $T: H^{s} \rightarrow H^{s-r}$ for each real $s$. We set $O P(-\infty)=\bigcap_{t>0} O P(-t)$.

Definition. $T \in N K(r)$ if and only if $T \in O P(r)$ and there is a $\sigma>0$ such that $\left[\ldots\left[\left[T, \varphi_{1}\right], \varphi_{2}\right] \ldots \varphi_{j}\right] \in O P(r-j \sigma)$ if $\varphi_{k} \in C_{0}^{\infty}\left(R^{n}\right)$. (Note that $T \in N K(r)$ implies $[T, \varphi] \in N K(r-\sigma)$ if $\varphi \in C_{0}^{\infty}$.) We shall set $P D(r)=P S(r) \cap N K(r)$.

Proposition 4. If $T \in N K(r)$ and if $\varphi, \psi \in C_{0}^{\infty}$ are such that supp $\varphi \cap \operatorname{supp} \psi=\varnothing$, then $\varphi T \psi \in O P(-\infty)$. Hence $T$ has a kernel which is $C^{\infty}$ off the diagonal.

Proof. $\varphi T \psi=T \varphi \psi-[T, \varphi] \psi=-[T, \varphi] \psi \in O P(r-\sigma)$. If $\varphi_{1}, \psi_{1} \in C_{0}^{\infty}$ are taken such that $\varphi_{1}=1$ in a neighborhood of $\operatorname{supp} \varphi, \psi_{1}=1$ in a neighborhood of supp $\psi$, and $\operatorname{supp} \varphi_{1} \cap \operatorname{supp} \psi_{1}=\varnothing$, then we can repeat this argument to get $\varphi T \psi$ $=\varphi_{1}(\varphi T \psi) \psi_{1} \in O P(r-2 \sigma)$. Continuing in this fashion, we have $\varphi T \psi \in O P(r-j \sigma)$ for each $j$, and hence $\varphi T \psi \in O P(-\infty)$.

Of course, we would like to know that $P D(r)$ contains lots of operators. This we establish in the next few propositions.

Proposition 5. $N K(r)$ is a linear space. If $T_{1} \in N K(r)$ and $T_{2} \in N K\left(r^{\prime}\right)$, then $T_{1} T_{2} \in N K\left(r+r^{\prime}\right)$.

Proof. The first assertion is trivial. As for the second, we have the formula $\left[T_{1} T_{2}, \varphi\right]=T_{1}\left[T_{2}, \varphi\right]+\left[T_{1}, \varphi\right] T_{2} \in O P\left(r+r^{\prime}-\sigma\right)$. Each term is the product of elements 
of $N K(t)$ for some appropriate $t$, so the reasoning above can be repeated for [[ $\left.\left.T_{1} T_{2}, \varphi_{1}\right], \varphi_{2}\right]$, etc., and our theorem follows by induction.

Proposition 6. $Q^{s} \in N K(2 s)$ for all real s. We can take $\sigma=2 \rho / \nu$ where $\nu$ is the order of $Q(D)$ and $\rho$ is such that $\left|Q^{(\alpha)}(\xi) / Q(\xi)\right| \leqq C|\xi|^{-\rho|\alpha|}$ for $|\xi|$ large.

Proof. Proposition 1 of $\S \mathrm{I}$ derives the formula, when $s=-t<0$,

$\left[Q^{-t}, \varphi\right]=\sum_{j=1}^{m-1} \sum_{\alpha_{1}, \ldots, \alpha_{j} \neq 0} w_{j, \alpha_{1}, \ldots, \alpha_{j}}(x) Q^{\left(\alpha_{1}\right)}(D) \cdots Q^{\left(\alpha_{j}\right)}(D) Q^{-t-j} \bmod O P(-2 m)$, with $w_{j, \alpha_{1}, \ldots, \alpha_{j}} \in C_{0}^{\infty}\left(R^{n}\right)$. Keeping in mind the formula

$$
\left[\prod_{j=1}^{N} X_{j}, Y\right]=\sum_{j=1}^{N}\left(\prod_{k=1}^{j-1} X_{k}\right)\left[X_{j}, Y\right]\left(\prod_{k=j}^{N} X_{k}\right),
$$

we see that $\left[\cdots\left[\left[Q^{-t}, \varphi_{1}\right], \varphi_{2}\right] \cdots \varphi_{j}\right]$ has a similar expression, where the sum belongs to $O P(-2 t-j \sigma)$, so taking $m$ large enough we deduce that this multiple commutator belongs to $O P(-2 t-j \sigma)$. This takes care of the case $s<0$. On the other hand, Leibniz' formula immediately implies $Q \in N K(2)$, so by Proposition $5, Q^{k} \in N K(2 k)$. Hence if $s>0$ and if $k>s$ is an integer, we have $Q^{s}=Q^{k} Q^{-(k-s)} \in N K(2 s)$.

Proposition 7. If $q(D)<P(D)$ then $q(D) \in N K(1)$. We can take $\sigma=\rho / \nu$ where $\nu$ is the order of $P(D)$ and $\rho$ is such that $\left|P^{(\alpha)}(\xi) / P(\xi)\right| \leqq C|\xi|^{-\rho|\alpha|}$ for $|\xi|$ large.

Proof. This is a simple consequence of the Leibniz formula, together with Lemma 1 of $\S \mathbf{I}$.

Proposition 8. $\mathfrak{A}_{0} \subset P D(0)$.

Proof. This is an obvious consequence of the three preceding propositions.

The following theorem explains the main reason why $P D(r)$ is interesting.

THEOREM 6. If $T \in P D(r)$ and $\sigma_{r}(T) \neq 0$ on $S$, and if $V \in P S(-r)$ is an operator such that $T V-I$ and $V T-I$ belong to PS $(-\infty)$ (such $V$ exist by Theorem 5$)$ then $V \in P D(-r)$.

Proof. If $V T=I+K_{1}$ and $T V=I+K_{2}$ with $K_{f} \in P S(-\infty)$, then

$$
\begin{aligned}
{[V, \varphi] } & =[V, \varphi]\left(T V-K_{2}\right)=(V \varphi-\varphi V) T V-[V, \varphi] K_{2} \\
& =(V \varphi T-\varphi V T) V-[V, \varphi] K_{2}=(V T \varphi-\varphi V T-V[T, \varphi]) V-[V, \varphi] K_{2} \\
& =-V[T, \varphi] V+K_{1} \varphi-\varphi K_{1}-[V, \varphi] K_{2}=-V[T, \varphi] V+K^{\prime}
\end{aligned}
$$

with $K^{\prime} \in P S(-\infty)$. From this it follows easily by induction that

$$
\left[\ldots\left[\left[V, \varphi_{1}\right], \varphi_{2}\right] \ldots \varphi_{j}\right] \in O P(-r-j \sigma) .
$$

From this one can by a well-known argument deduce again the local regularity theorem for a formally hypoelliptic differential operator of constant strength. 
IV. Hypoelliptic operators of constant strength. In his thesis, L. Hörmander gave a necessary and sufficient condition that an operator with constant coefficients be hypoelliptic. Later, Hörmander [6], and, independently, Malgrange [7] showed that a differential operator $p(x, D)$ of constant strength such that the constant coefficients operator $p\left(x_{0}, D\right)$ satisfies this condition is hypoelliptic. We complete this circle of theorems by showing that if $p(x, D)$ has constant strength and is hypoelliptic, then so is $p\left(x_{0}, D\right)$.

LEMMA. Let $p(x, D)$ be a differential operator of constant strength on a domain $\Omega$. Let $x_{0} \in \Omega, P_{0}(D)=p\left(x_{0}, D\right)$. If $\varepsilon>0$ is given, there is a neighborhood $\omega$ of $x_{0}, a$ continuous operator $T$ on $L^{2}(\omega)$, and a differential operator $Q(D)$ which does not depend on $\omega$ or $T$, such that

(i) $Q(D)$ and $P_{0}(D)$ have the same strength,

(ii) $p(x, D) u=Q(D) T u$ for all $u \in L^{2}(\omega)$,

(iii) $\left\|I-T^{-1}\right\|<\varepsilon$.

Proof. By Theorem 7.1.1 of [4], we can write $p(x, D)^{t}=\bar{Q}(D)+\sum_{j=1}^{N} d_{j}(x) R_{j}(D)$ where $Q(D)$ has the same strength as $P_{0}(D)$, each $R_{j}(D)$ is weaker than $Q(D)$, and $d_{j}\left(x_{0}\right)=0$. Now if $E_{0}$ is Hörmander's fundamental kernel for the operator $\bar{Q}(D)$, then, as shown in Theorem 7.2.1 of [4], the operator $A$ given by $A g=$ $\sum d_{j}(x) R_{j}(D) E_{0} g$ is a bounded operator on $L^{2}(\omega)$, and if $\omega$ is chosen small enough, $\|A\|<\varepsilon_{1}$; also if $E=E_{0}(I+A)^{-1}$, then $p(x, D)^{t} E=I$ on $L^{2}(\omega)$, and $E p(x, D)^{t} v$ $=v$ if $v \in C_{0}^{\infty}(\omega)$.

Applying $\bar{Q}(D)$ to both sides of the equality $E=E_{0}(I+A)^{-1}$, we get $\bar{Q}(D) E$ $=(I+A)^{-1}$, so $\bar{Q}(D) E p(x, D)^{t} v=(I+A)^{-1} p(x, D)^{t} v$ if $p(x, D)^{t} v \in L^{2}(\omega)$, and in particular if $v \in C_{0}^{\infty}(\omega)$. Since $E p(x, D)^{t} v=v$ in this case, we see that $\bar{Q}(D) v$ $=(I+A)^{-1} p(x, D)^{t} v$ if $v \in C_{0}^{\infty}(\omega)$. Now with arbitrary $v \in C_{0}^{\infty}(\omega)$ and arbitrary $u \in L^{2}(\omega)$, if ( , ) denotes the inner product in $L^{2}(\omega)$ and also between elements of $C_{0}^{\infty}(\omega)$ and distributions on $\omega$, taken to be linear in the first argument and conjugate linear in the second, we have

$$
\begin{aligned}
(Q(D) u, v) & =(u, \bar{Q}(D) v)=\left(u,(I+A)^{-1} p(x, D)^{t} v\right)=\left(\left(I+A^{*}\right)^{-1} u, p(x, D)^{t} v\right) \\
& =\left(p(x, D)\left(I+A^{*}\right)^{-1} u, v\right),
\end{aligned}
$$

so for all $u \in L^{2}(\omega), Q(D) u=p(x, D)\left(I+A^{*}\right)^{-1} u$, as elements of $\mathscr{D}^{\prime}(\omega)$. We need only take $T=I+A^{*}$, and since $A$ can be taken arbitrarily close to $0, T$ can be taken arbitrarily close to $I$, insuring that $\left\|I-T^{-1}\right\|<\varepsilon$.

THEOREM. If $p(x, D)$ is a hypoelliptic differential operator on $\Omega$, and if it has constant strength, then $P_{0}(D)=p\left(x_{0}, D\right)$ is hypoelliptic.

Proof. We have just shown that there is a neighborhood $\omega$ of $x_{0}$, a continuous invertible operator $T$ on $L^{2}(\omega)$, and a differential operator $Q(D)$ of the same strength as $P_{0}(D)$ such that $p(x, D) u=Q(D) T u$ for all $u \in L^{2}(\omega)$, and we can assume that $\left\|I-T^{-1}\right\|$ is as small as desired. It will suffice to prove that $Q(D)$ is hypoelliptic, by Theorem 4.1 .6 of [4]. If not, there is a sequence $\zeta_{v} \in C^{n}$ such that $Q\left(\zeta_{v}\right)=0$ and 
$\left|\zeta_{\nu}\right| \rightarrow \infty$ while $\left|\operatorname{Im} \zeta_{\nu}\right|$ remains bounded. We now require that

$$
\left\|I-T^{-1}\right\| \leqq \inf _{v ; x \in \omega} \frac{1}{2} \exp \left(-2\left|\operatorname{Im} \zeta_{v}\right||x|\right) .
$$

Let $u_{v}(x)=\exp \left(i\left\langle x, \zeta_{v}\right\rangle\right)$ and $v_{v}=T^{-1} u_{v}$. Then $p(x, D) v_{v}=Q(D) T T^{-1} u_{v}=Q(D) u_{v}$ $=0$. Also $u_{v} \rightarrow 0$ weakly in $\mathscr{D}^{\prime}(\omega)$; indeed if $\varphi \in C_{0}^{\infty}(\omega)$ we have $\int \varphi u_{v} d x$ $=\hat{\varphi}\left(-\zeta_{v}\right) \rightarrow 0$ as $v \rightarrow \infty$, by the Paley-Wiener theorem. Since $\left\{u_{v}\right\}$ is a bounded subset of $L^{2}(\omega)$, it follows that $u_{v} \rightarrow 0$ weakly in $L^{2}(\omega)$. Hence $v_{v} \rightarrow 0$ weakly in $L^{2}(\omega)$.

Since $p(x, D)$ is hypoelliptic, $N=\operatorname{ker} p(x, D) \subset L_{\text {loc }}^{2}(\omega)$ is a Montel space; it coincides with $\operatorname{ker} p(x, D) \subset C^{\infty}(\omega)$ and the two induced topologies coincide on $\operatorname{ker} p(x, D)$ by the open mapping theorem for Fréchet spaces. Since $\left\{u_{v}\right\}$ is bounded in $L^{2}(\omega)$, so is $\left\{v_{v}\right\}$, and a fortiori $\left\{v_{v}\right\}$ is bounded in $L_{\text {loc }}^{2}(\omega)$. Since $N$ is a Montel space, $\left\{v_{v}\right\}$ has a convergent subsequence in $L_{\text {loc }}^{2}(\omega)$, which we continue to denote by $\left\{v_{v}\right\}$. Since $v_{v} \rightarrow 0$ weakly on $L^{2}(\omega)$, we have a fortiori $v_{v} \rightarrow 0$ weakly on $L_{\text {loc }}^{2}(\omega)$, so 0 can be the only limit point of $\left\{v_{v}\right\}$. Hence $v_{v} \rightarrow 0$ in $L_{\text {loc }}^{2}(\omega)$.

But if $\varphi \in C_{0}^{\infty}(\omega)$, we have

$$
\begin{aligned}
2\left\|\varphi v_{v}\right\|^{2} & \geqq\left\|\varphi u_{v}\right\|^{2}-2\left\|\varphi\left(I-T^{-1}\right) u_{v}\right\|^{2} \\
& \geqq \inf _{v: x \in \omega} \exp \left(-2\left|\operatorname{Im} \zeta_{v}\right||x|\right)\left[\|\varphi\|^{2}-\frac{1}{2}(\operatorname{vol} \omega)^{2}\right]
\end{aligned}
$$

if $\|\varphi(x)\| \leqq 1$ on $\omega$. But such a $\varphi \in C_{0}^{\infty}(\omega)$ can be found with $\varphi=1$ on a sufficiently large portion of $\omega$ that we get $\left\|\varphi v_{v}\right\|^{2} \geqq \delta>0$ for all $\nu$, a contradiction. This completes the proof.

REMARK. The above proof is a modification of the proof of Theorem 4.1.1 in [4].

\section{REFERENCES}

1. M. Breuer and H. Cordes, On Banach algebras with o-symbol, J. Math. Mech. 13 (1964), 313-324. MR 28 \#2456.

2. H. Cordes and E. Herman, Gelfand theory of pseudo differential operators, Amer. J. Math. 90 (1968), 681-717.

3. J. Dixmier, Les $C^{*}$-algebres et leurs répresentations, Cahiers Scientifiques, fasc. 29 , Gauthier-Villars, Paris, 1964. MR 30 \#1404.

4. L. Hörmander, Linear partial differential operators, Die Grundlehren der math. Wissenschaften, Band 116, Academic Press, New York; Springer-Verlag, Berlin, 1963. MR 28 \#4221.

5. - Pseudo-differential operators and hypoelliptic equations, Proc. Sympos. Pure Math., vol. 10, Amer. Math. Soc., Providence, R. I., 1967, pp. 138-183.

6. - On interior regularity of the solutions of partial differential equations, Comm. Pure Appl. Math. 11 (1958), 197-218. MR 21 \#5064.

7. B. Malgrange, Sur une classe d'opérateurs différentiels hypoelliptiques, Bull. Soc. Math. France 85 (1957), 283-306. MR 21 \#6063.

8. E. Nelson and W. F. Stinespring, Representation of elliptic operators in an enveloping algebra, Amer. J. Math. 81 (1959), 547-560. MR 22 \#907.

UNIVERSITY OF CALIForNIA,

Berkeley, California 94720 